\title{
Perfectly Matched Layer Based Modelling of Layered Media: Overview and Perspective
}

\author{
Dries Vande Ginste ${ }^{\# 1}$, Hendrik Rogier \#2, Daniël De Zutter \#3 \\ \# Electromagnetics Group, Department of Information Technology, Ghent University \\ Sint-Pietersnieuwstraat 41, B-9000 Gent, Belgium \\ ${ }^{1}$ dries.vandeginste@intec.UGent.be \\ 2 hendrik.rogier@intec.UGent.be \\ ${ }^{3}$ daniel.dezutter@intec.UGent.be
}

\begin{abstract}
Whereas the Perfectly Matched Layer (PML) was originally conceived to serve as a reflectionless absorbing boundary condition, terminating the infinite simulation domain in finite difference or finite element electromagnetic field solvers, unexpected applications of the PML arose by using it to close down open layered waveguide configurations. As a tribute to our former colleague, Prof. F. Olyslager, in this contribution, the PML-paradigm for layered media is explained and an overview of this paradigm's applications is presented. A novel illustrative example, focussing on the modelling of periodic microstrip structures, is also provided.
\end{abstract}

\section{INTRODUCTION}

The Perfectly Matched Layer (PML) was originally conceived by J.-P. Bérenger [1] to serve as an absorbing boundary condition in finite-difference time-domain (FDTD) solvers. Bérenger presented the PML via a split field formalism, leading to a reflectionless absorption of incident waves arriving from all angles and for all frequencies. Later, it was found that the PML could be physically interpreted as an artificial uniaxial anisotropic medium [2]. A very elegant description of the PML, described first by Chew and Weedon [3], is based on complex coordinate stretching. This latter description allows to treat a PML in the frequency domain as a homogenous, isotropic layer with a complex thickness.

The idea of using the PML as an absorbing layer to terminate infinite domains led to efficient and elegant modelling techniques in a completely different context. Many waveguides geometries consist of an open stack of dielectric layers. The modal spectra of these open waveguides comprise at least one continuous set of radiation modes. The integral over all modes must be calculated to completely describe the fields. Consequently, efficient modelling of open waveguides is not at all straightforward. The new PML-paradigm, mathematically detailed by F. Olyslager in [4], allows to convert the open stack of dielectric layers into a closed waveguide. The presence of the PML ensures that the closed waveguide accurately mimics the behavior of the original open waveguide. The modal spectrum of the closed waveguide only exhibits discrete sets of modes.

In this contribution the PML-paradigm is explained and an overview of modelling techniques, evolving from this paradigm and developed under the supervision of, a.o., Prof. F. Olyslager, is given (Section II). Our most recent development concerns the modelling of periodic metallizations residing in planarly layered background media. There is a broad range of applications for this kind of structures. Hence, in Section III, a brief introduction to PML-based modeling of one-dimensional (1-D) periodic microstrip structures is presented. Conclusions are summarized in Section IV.

\section{THE PML-PARADIGM: THEORY AND APPLICATIONS}

\section{A. Closure of open waveguides}

Many waveguide configurations consist of an open stack of dielectric layers. To solve wave equations corresponding to these structures using an eigenmode expansion technique, classically one has to deal with one or more continuous sets of radiation modes appearing in the modal spectrum of the waveguides. Consider, e.g., the microstrip structure comprising a dielectric slab residing on a perfect electrically conducting (PEC) ground plate, shown in Fig. 1(a), and the optical fiber consisting of two dielectric layers, i.e. the core and the cladding, presented in Fig. 1(c). In both these cases, there is one such set of radiation modes, corresponding to the air halfspace above the microstrip or the air surrounding the fiber. Open waveguides can be closed by means of a PML backed by a PEC wall. Using the complex coordinate stretching formalism [3], it is shown that this procedure is mathematically equivalent to (i) placing a PEC plate at a complex distance $\mathcal{D}$ above the dielectric slab (see Fig. 1(b)) or (ii) enclosing the optical fiber in a PEC cylinder with complex radius $\mathcal{D}$ (see Fig. 1(d)). In all cases, the complex value of $\mathcal{D}$ must be chosen such that the PML provides damping along the direction perpendicular to the layer interfaces, i.e. the direction $z$ in case of the microstrip or the radial direction $\rho=\sqrt{x^{2}+y^{2}}$ in case of the fiber. Adopting a time-harmonic $e^{j \omega t}$ dependency of the fields, where $\omega$ is the angular frequency, this necessary damping is achieved when the real part $\Re(\mathcal{D})>0$ and the imaginary part $\Im(\mathcal{D})<0$. Due to the reflectionless absorption of waves impinging upon the PML, PEC-backed PML-closed structures (e.g. Figs. 1(a) and 1(c)) closely mimic the behavior of the original, open structures (e.g. Figs. 1(b) and 1(d)). However, the original modal spectrum is now replaced by a discrete set of modes of the PEC-backed PML-closed waveguide. This is illustrated in Figs. 1(e) and 1(f), showing the location of the propagation constants $\beta$ of these modes in the complex 


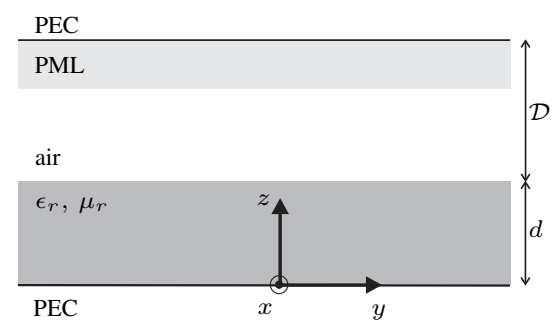

(a) open microstrip substrate

(b) PEC-backed PML-closed microstrip substrate

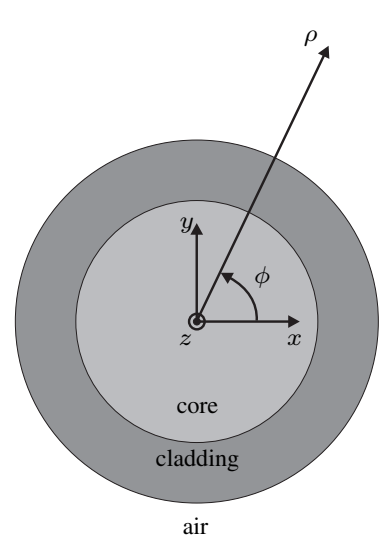

(c) open optical fiber

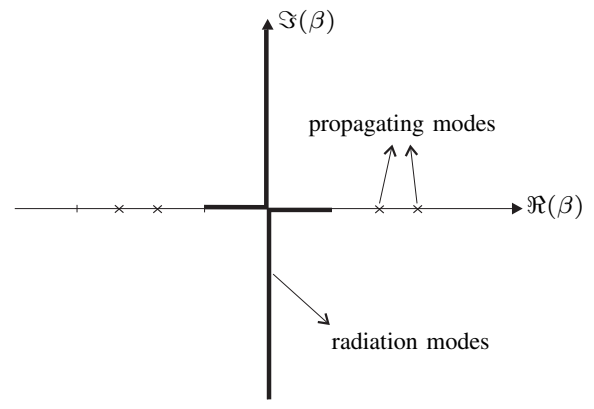

(e) continuous spectrum (proper Riemann sheet)

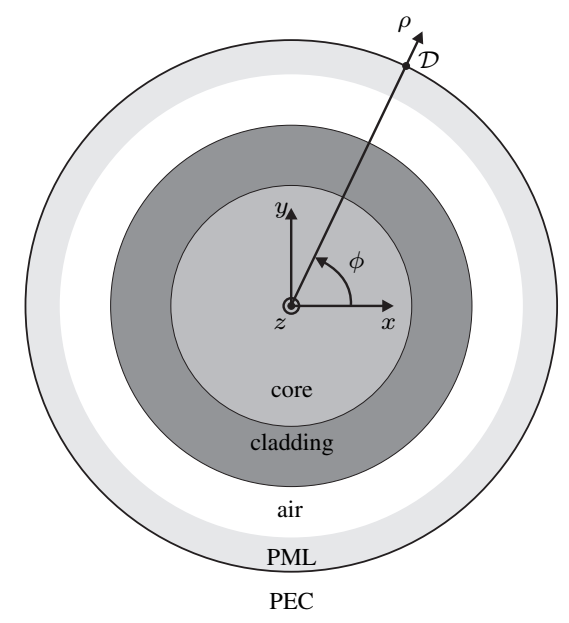

(d) PEC-backed PML-closed optical fiber

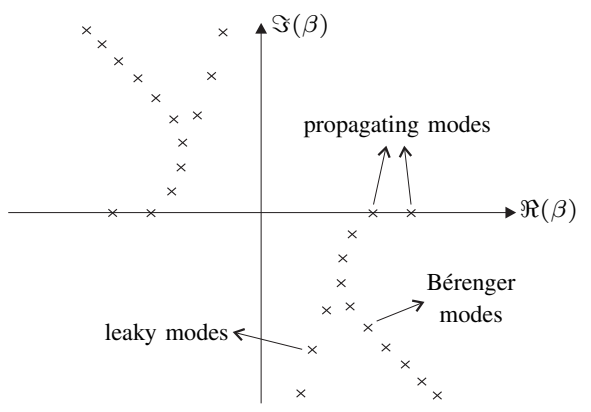

(f) discrete spectrum (proper Riemann sheet)

Fig. 1. Closure of open planarly and circularly layered waveguides and corresponding modal spectra

$\beta$-plane. Typically, the PML-eigenmodes (Fig. 1(f)) come in three flavors. First, there are the propagating modes that are to a high degree of accuracy identical to those found in the original open waveguide. Second, there are the leaky modes. The field behavior of these modes inside the dielectric layers is very similar to that of leaky modes in the original, open waveguide. These latter are not shown in Fig. 1(e) as for open waveguides, the propagation constants of these modes are found as improper solutions of the pertinent dispersion relation, where the term improper is used to indicate that these modes do not satisfy the radiation condition [5]. For PECbacked PML-closed waveguides, the exponential increase of the field of these eigenmodes is suppressed by entering into the complex plane. Third, there are the so-called Bérenger modes, featuring an exponentially decreasing, oscillatory behavior in the PML-layer. Together, these propagating, leaky, and Bérenger modes form a complete set of the PEC-backed PMLclosed waveguide [4], [6].

\section{B. Overview of applications}

The replacement of the radiation modes by a discrete set of modes allows the expansion of the fields into rapidly convergent series of eigenmodes. We can distinguish between two categories: (i) mode matching techniques and (ii) Green's functions based integral equation techniques.

- First, the mode matching technique was developed for the analysis of semi-conductor laser-facets [7], immediately revealing the potential of this approach. This mode matching technique for planarly layered media 


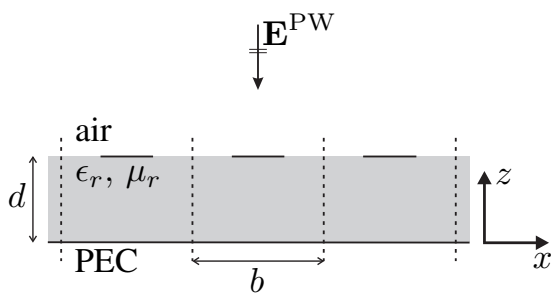

(a) Frontal view

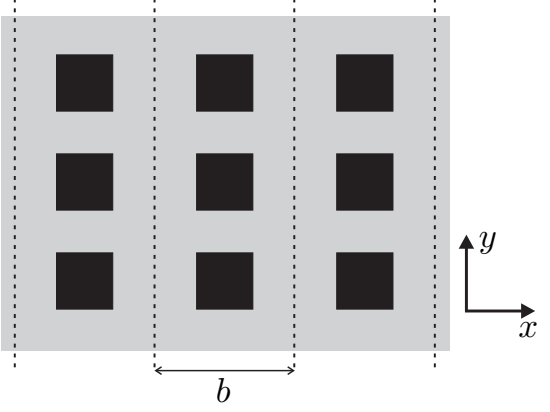

(b) Top view

Fig. 2. Geometry of a 1-D periodic microstrip array

was further developed for both microwave and optical devices [8]-[10]. Interestingly, the acoustics community also picked up the formalism and the PML-paradigm for planarly layered media was applied to the Pekeris waveguide [11]. Cylindrical dielectric waveguides, such as optical fibers and vertical-cavity surface-emitting lasers (VCSELs), are of particular importance in photonics applications and eigenmode expansion techniques for these kind configurations have also been conceived [12], [13]. It is well-known that for mode matching techniques to be accurate, a large number of modes needs to be retained. Therefore, a lot of research has been and still is devoted to the efficient computation of many modes of (multi)layered planar and circular dielectric waveguides [14]-[17].

- Full-wave microwave planar circuit simulators often rely on boundary integral equation (BIE) techniques combined with a method of moments (MoM) [18]. Many of these formalisms use layered medium Green's functions and therefore only require discretization of the circuit's finite metallic conductors. Assuming their currents are represented in terms of $N$ basis functions, these solvers call for the direct or iterative solution of a dense $N \times N$ matrix system of linear equations. Unfortunately, construction of this system requires the calculation of layered medium Green's functions [19], which necessitates the time-consuming numerical evaluation of order $\mathcal{O}\left(N^{2}\right)$ Sommerfeld-type integrals [20]. This is due to the continuous set of radiation modes, appearing as a branch cut (see Fig. 1(e)) in the modal spectrum of the spectral Green's functions. Therefore, new algorithms have been developed leveraging PMLs to represent open layered medium Green functions in terms of an exponentially convergent series of discrete eigenmodes of the PEC-backed PML-closed waveguide [4], [21]-[23]. This closed-form representation of the pertinent Green's function leads to efficient and very accurate modelling techniques. Furthermore, it has been shown that a MoM based on the new series can be accelerated by means of the Fast Multipole Method [24], yielding modelling techniques with $\mathcal{O}(N)$ computational cost and a controllable accuracy [25]-[28].

\section{PML-BASED MODELLING OF 1-D PERIODIC MICROSTRIP STRUCTURES}

The study of periodic structures residing in layered background media has been a research topic for many years. The range of applications comprises antenna arrays, electromagnetic bandgap structures, frequency selective surfaces, leakywave antennas, etc. The periodic character of these structure can be exploited by using the Floquet-Bloch theorem, allowing to restrict the analysis of the complete structure (with infinite extent) to one representative unit cell. Similarly to what is described in Section II-B for non-periodic planarly layered media, BIE techniques can be conceived provided that the pertinent periodic Green's functions can be efficiently determined. Again, the PML-paradigm was successfully applied to determine periodic layered medium Green's functions with 1-D periodicity in closed-form for 2-D [29] and 3-D [30] configurations. Our current research focusses on the application of these exponentially convergent series in novel simulation techniques. As a first step, consider a microstrip patch array with 1-D periodicity, as shown in Fig. 2. The microstrip substrate has a thickness $d=3.17 \mathrm{~mm}$, a relative permittivity $\epsilon_{r}=11.7$, and a relative permeability $\mu_{r}=1$. One unit cell consist of three patches of dimension $10 \mathrm{~mm} \times 10 \mathrm{~mm}$. The period is $b=40 \mathrm{~mm}$ and the spacing between the patches along the $y$ direction is $10 \mathrm{~mm}$. The angular frequency is $\omega=2 \pi 10 \mathrm{GHz}$. The structure is illuminated by a plane wave $\mathbf{E}^{\mathrm{PW}}(\mathbf{r})=$ $e^{j k_{0} z} \hat{\mathbf{y}}$. The $y$-component of the current density, denoted $J_{y}$, induced by this plane wave, is calculated by means of the new 1-D periodic BIE technique and compared with the solution of a reference program, the SVD-PML-MLFMA for finite, nonperiodic structures [27]. With the SVD-PML-MLFMA a finite array of $7 \times 3$ patches, i.e. seven unit cells, is simulated and the current flowing on the central unit cell is used as a reference result. Both currents are plotted in Fig. 3. Although only seven periods were used in the reference program, good agreement can already be observed, as such validating the approach and showing a promising perspective for further research. It is interesting to mention that, for the example of Fig. 3, the total solution time using the new 1-D periodic BIE technique is about 56 times smaller than when using the SVD-PMLMLMFA. This is due to the small system matrix. 


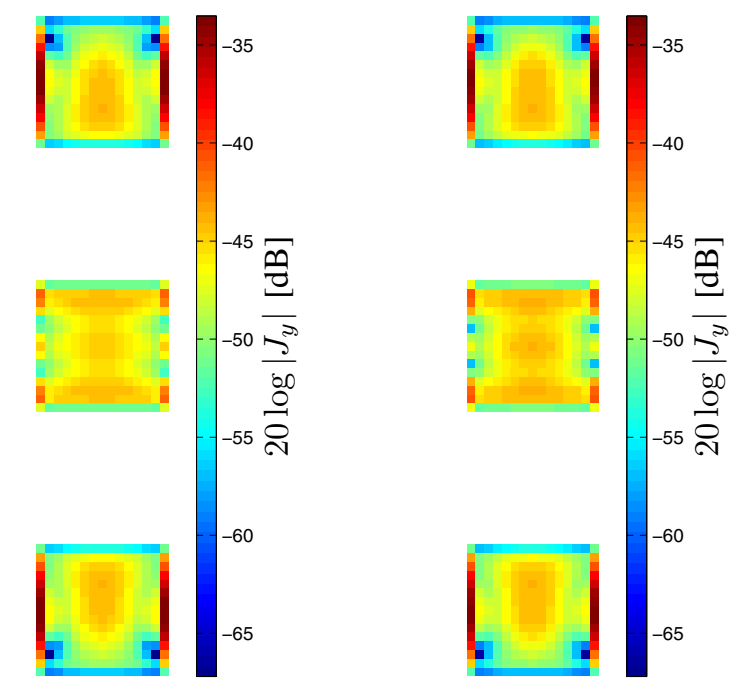

Fig. 3. Comparison of the $y$-oriented current flowing within one unit cell of an array of patches. Left: results obtained via the PML-based periodic Green's functions. Right: results obtained using the SVD-PML-MLFMA [27].

\section{CONCLUSION}

During recent years, apart from serving as absorbing boundary conditions in FDTD methods, PMLs were used to terminate open layered waveguide configurations. In this contribution, the PML-paradigm, allowing for the closure of open layered media, is briefly summarized and an overview is given of new modelling techniques evolving from this approach. A perspective for further research, i.e. the efficient modelling of periodic metallizations residing in planarly layered background media, is described. A first illustrative example has also been provided.

\section{REFERENCES}

[1] J. P. Bérenger, "A perfectly matched layer for the absorption of electromagnetic waves," Journal of Computational Physics, vol. 114, no. 2, pp. 185-200, Oct. 1994.

[2] Z. S. Sacks, D. M. Kingsland, R. Lee, and J. F. Lee, "A perfectly matched anisotropic absorber for use as an absorbing boundary condition," IEEE Trans. Antennas Propag., vol. 43, pp. 1460-1463, Dec. 1995.

[3] W. C. Chew and W. H. Weedon, "A 3D perfectly matched medium from modified Maxwell's equations with stretched coordinates," Microwave Opt. Technol. Lett., vol. 7, no. 13, pp. 599-604, Sept. 1994.

[4] F. Olyslager, "Discretization of continuous spectra based on perfectly matched layers," SIAM J. Appl. Math., vol. 64, no. 4, pp. 1408-1433, May 2004.

[5] J. Van Bladel, Electromagnetic Fields, 2nd ed. Piscataway, NJ, USA: IEEE Press, 2007.

[6] L. F. Knockaert and D. De Zutter, "On the completeness of eigenmodes in a parallell plate waveguide with a perfectly matched layer termination," IEEE Trans. Antennas Propag., vol. 50, no. 11, pp. 1650-1653, Nov. 2002.

[7] H. Derudder, D. De Zutter, and F. Olyslager, "Analysis of waveguide discontinuities using perfectly matched layers," Electronics Letters, vol. 34, no. 22, pp. 2138-2140, Oct. 1998.

[8] P. Bienstman and R. Baets, "Advanced boundary conditions for eigenmode expansion models," Optical and Quantum Electronics, vol. 34, no. 5, pp. 523-540, May-June 2002.

[9] H. Derudder, F. Olyslager, and D. De Zutter, "Efficient calculation of far-field patterns of waveguide discontinuities using perfectly matched layers," Electronics Letters, vol. 36, no. 9, pp. 798-799, Apr. 2000.
[10] H. Derudder, F. Olyslager, D. De Zutter, and S. Van den Berghe, "Efficient mode-matching analysis of discontinuities in finite planar substrates using perfectly matched layers," IEEE Trans. Antennas Propag., vol. 49, no. 2, pp. 185-195, Feb. 2001.

[11] J. Zhu and Y. Y. Lu, "Asymptotic solutions of the leaky modes and PML modes in a Pekeris waveguide," Wave Motion, vol. 45, no. 3, pp. 207-216, Jan. 2008.

[12] P. Bienstman, H. Derudder, R. Baets, F. Olyslager, and D. De Zutter, "Analysis of cylindrical waveguide discontinuities using vectorial eigenmodes and perfectly matched layers," IEEE Trans. Microwave Theory Tech., vol. 49, no. 2, pp. 349-354, Feb. 2001.

[13] P. Bienstman, E. Six, M. Roelens, M. Vanwolleghem, and R. Baets, "Calculation of bending losses in dielectric waveguides using eigenmode expansion and perfectly matched layers," IEEE Photonics Technology Letters, vol. 14, no. 2, pp. 164-166, Feb. 2002.

[14] H. Rogier and D. De Zutter, "Bérenger and leaky modes in microstrip substrates terminated by a perfectly matched layer," IEEE Trans. Microwave Theory Tech., vol. 49, no. 4, pp. 712-715, April 2001.

[15] H. Rogier and D. Vande Ginste, "A fast procedure to accurately determine leaky modes in multilayered planar dielectric substrates," IEEE Trans. Microwave Theory Tech., vol. 56, no. 6, pp. 1413-1422, June 2008.

[16] H. Rogier and D. De Zutter, "Berenger and leaky modes in optical fibers terminated with a perfectly matched layer," IEEE J. Lightwave Technol., vol. 20, no. 7, pp. 1141-1148, Jul. 2002.

[17] D. Vande Ginste, H. Rogier, and D. De Zutter, "Efficient computation of TM- and TE-polarized leaky modes in multilayered circular waveguides," Submitted to Journal of Lightwave Technology.

[18] R. F. Harrington, Field computation by moment methods. Piscataway, NJ: IEEE Press, 1993.

[19] K. A. Michalski and D. Zheng, "Electromagnetic scattering and radiation by surfaces of arbitrary shape in layered media, Part I: Theory," IEEE Trans. Antennas Propag., vol. 38, no. 3, pp. 335-344, March 1990.

[20] N. Faché, F. Olyslager, and D. De Zutter, Electromagnetic and Circuit Modelling of Multiconductor Transmission Lines. New York: Oxford University Press Inc., 1993.

[21] H. Derudder, F. Olyslager, and D. De Zutter, "An efficient series expansion for the 2D Green's function of a microstrip substrate using perfectly matched layers," IEEE Microwave Guided Wave Lett., vol. 9, no. 12, pp. 505-507, Dec. 1999.

[22] H. Derudder, D. De Zutter, and F. Olyslager, "Efficient calculation of the 2D Green's function of a truncated grounded dielectric slab," IEEE AP-S Int. Symp., vol. 2, pp. 618-621, July 2000.

[23] F. Olyslager and H. Derudder, "Series representation of Green dyadics for layered media using PMLs," IEEE Trans. Antennas Propag., vol. 51, no. 9, pp. 2319-2326, Sept. 2003.

[24] W. C. Chew, Waves and fields in inhomogeneous media. New York: Van Nostrand Reinhold, 1990, reprinted by IEEE press, 1995.

[25] D. Vande Ginste, H. Rogier, D. De Zutter, and F. Olyslager, "A fast multipole method for layered media based on the application of perfectly matched layers - the 2-D case," IEEE Trans. Antennas Propag., vol. 52, no. 10, pp. 2631-2640, Oct. 2004.

[26] D. Vande Ginste, E. Michielssen, F. Olyslager, and D. De Zutter, "An efficient perfectly matched layer based multilevel fast multipole algorithm for large planar microwave structures," IEEE Trans. Antennas Propag., vol. 54, no. 5, pp. 1538-1548, May 2006.

[27] _ "A high-performance upgrade of the perfectly matched layer multilevel fast multipole algorithm for large planar microwave structures," IEEE Transactions on Antennas and Propagation, vol. 57, no. 6, pp. 1728-1739, June 2009.

[28] D. Vande Ginste, L. Knockaert, and D. De Zutter, "Error control in the perfectly matched layer based multilevel fast multipole algorithm," Journal of Computational Physics, vol. 228, no. 13, pp. 4811-4822, July 2009.

[29] H. Rogier and D. De Zutter, "A fast converging series expansion for the 2-D periodic Green's function based on perfectly matched layers," IEEE Trans. Microwave Theory Tech., vol. 52, no. 4, pp. 1199-1206, April 2004.

[30] H. Rogier, "New series expansions for the 3D Green's function of multilayered media with 1D periodicity based on perfectly matched layers," IEEE Trans. Microwave Theory Tech., vol. 55, no. 8, pp. 1730-1738, Aug. 2007. 